\title{
Vers une Société Française de Physique plus efficace
}

La physique, discipline centrale de notre connaissance de la nature, est au cœur d'un enjeu majeur dans le monde d'aujourd'hui, celui de l'innovation, de la recherche, du développement scientifique et technique, de la formation des cadres scientifiques du plus haut niveau et, finalement, de l'attractivité de notre pays. La physique est ainsi une des principales clés de l'adaptation à la compétition mondiale de nos industries, de nos capacités de progrès technologique dans pratiquement tous les champs disciplinaires, de la médecine aux sciences de I'ingénieur, du spatial aux nanotechnologies, du nucléaire au solaire, mais aussi de la technologie à la philosophie, de l'industrie à l'art. Elle est la discipline du $21^{\text {e }}$ siècle partout présente, à chaque instant, dans le quotidien de chacun d'entre nous.

Pourtant, malgré son importance et ses succès récents en matière de reconnaissance internationale, la physique hérite en France d'une situation paradoxale. Son poids relatif dans l'enseignement est en décroissance régulière. Ceci commence au lycée où le volume horaire des cours de physique diminue, et se poursuit à l'Université où le nombre d'étudiants baisse de manière inquiétante. De leur côté, la majorité des grandes écoles privilégie d'autres domaines plus directement adaptés à l'industrie ou à l'économie. Pour couronner le tout, la gestion actuelle de la recherche favorise les projets à caractère soi-disant finalisé, désavantageant ainsi la prise de risque indispensable à la production de ruptures technologiques majeures.

Créée en 1873, la Société Française de Physique (SFP) aurait dû avoir comme vocation de couvrir l'ensemble des sous-disciplines dérivées. Historiquement, ce ne fut pas le cas, avec comme résultat un affaiblissement de l'audience des physiciens. L'un de nos objectifs est donc de nous concerter avec les autres sociétés savantes qui nous sont proches, comme I'Union des Professeurs de Physique et de Chimie, par exemple. Ceci devrait être facilité par le fait que les divisions thématiques et les commissions de la SFP couvrent un large spectre, incluant souvent celui de ces sociétés.

Entrées dans un processus de refonte, les instances de la SFP ont la volonté de faire évoluer cette situation de manière à rendre plus audible la voix des physiciens. Pour ce faire, plusieurs actions sont en cours :

- Refonte totale du site web, avec externalisation des aspects techniques et mise sur pied d'une équipe interne susceptible de l'alimenter efficacement.

- Utilisation de ce site pour mieux coordonner et valoriser les actions des sections locales, des divisions thématiques et des commissions, dont les travaux et les réalisations sont considérables et pas suffisamment visibles.

- Gestion des congrès labellisés SFP à partir du nouveau site web.

- Incitation à l'implication dans la vie de la SFP de tous les acteurs de la recherche et de l'enseignement de la physique, à commencer par les étudiants et les jeunes chercheurs.

- Mise en valeur de notre revue Reflets de la Physique, vecteur de notre communication.

- Rapprochement avec les organismes de recherche, déjà largement entamé avec l'Institut de Physique du CNRS.

- Renforcement de nos relations avec les industriels, avec la nomination de Marc Stehle à la tête de la commission correspondante.

Nous espérons que tout ceci confortera la position des physiciens dans la société française actuelle.

Gérard Bonneaud

Secrétaire général
Michel Lannoo

Président 